\title{
Transmit Antenna Selection Aided Linear Group Precoding for Massive MIMO Systems
}

\author{
Van Khoi Dinh ${ }^{1, *}$, Minh Tuan $\mathrm{Le}^{2}$, Vu Duc Ngo ${ }^{3}$, Xuan Nam $\operatorname{Tran}^{1}$ and Chi Hieu Ta ${ }^{1}$ \\ ${ }^{1}$ Le Quy Don Technical University, Vietnam \\ ${ }^{2}$ MobiFone R\&D Center, MobiFone Corporation, Vietnam \\ ${ }^{3}$ Hanoi University of Science and Technology, Vietnam
}

\begin{abstract}
In this paper, we investigate the combination of linear group precoding with a transmit antenna group selection (TA-GS) algorithm based on the channel capacity analysis for Massive MIMO systems. Simultaneously, we propose a low complexity linear precoding algorithm that works on the selected antennas. The proposed precoder is developed based on the conventional linear precoders in combination with the element-base lattice reduction shortest longest vector technique having low complexity. Numerical and simulation results show that the system performance significantly improves when the transmit selection technique is applied. Besides, the proposed precoder has remarkably lower computational complexity than its LC-RBD-LR-ZF counterpart. The bit error rate (BER) performance of the proposed precoder can approach that of the LC-RBD-LR-ZF precoder as the number of groups increases, yet at the cost of higher detection complexity.
\end{abstract}

Keywords: MU-MIMO system, Massive MIMO system, linear precoder, lattice reduction algorithm.

Received on 13 September 2019, accepted on 11 October 2019, published on 24 October 2019

Copyright (C) 2019 Van Khoi Dinh et al., licensed to EAI. This is an open access article distributed under the terms of the Creative Commons Attribution licence (http://creativecommons.org/licenses/by/3.0/), which permits unlimited use, distribution and reproduction in any medium so long as the original work is properly cited.

doi: 10.4108/eai.24-10-2019.160982

${ }^{*}$ Corresponding author. Email: Vankhoi.tcu@gmail.com

\section{Introduction}

Multiuser Multiple-Input Multiple-Output (MUMIMO) technique has widely been studied for many years and is becoming a mature technology [1], [2]. In reality, the number of antennas at each BS in the MU-MIMO systems is usually small [3]. Therefore, the spectrum efficiency and system capacity are still relatively modest. This is one of the limitations of the MU-MIMO systems.

In order to cope with these issues, Massive MIMO systems have recently been proposed in [1], [4], [5], [6]. In the Massive MIMO systems, a Base Station (BS) uses large antenna arrays to serve several tens of users (or more) in the same time-frequency resources. The Massive MIMO systems can significantly improve the channel capacity, enhance the spectrum utilization efficiency and the system quality [5]. Basically, a Massive MIMO system can work in Time Division Duplex (TDD) or Frequency Division Duplex (FDD) mode. However, the
TDD-based Massive MIMO systems is preferable to the FDD-based Massive MIMO systems they relax the limitation of the number of antennas at the BSs [1]. It is expected that Massive MIMO will be a potential technique for the next generation mobile networks (e.g., 5G network) [1], [5], [7].

In Massive MIMO systems, the complex signal processing is performed at the BS side. Therefore, the precoding algorithms with low-complexity, such as Zero Forcing (ZF), Minimum Mean Square Error (MMSE) and Maximum Ratio Transmission (MRT), are considered as suitable solutions for the downlink in the Massive MIMO system [8], [9], [10]. In [11], the authors proposed the precoding algorithms according to group based on QR decomposition of the channel matrix and the Pseudo Inverse Block Diagonalization (PINV-BD) for Massive MIMO systems. In this proposal, the lattice reduction (LR) technique and Tomlinson-Halashima precoder (THP) algorithm are applied to each group to improve the system performance. In [12], $\mathrm{Zu}$ et al. proposed the Block 
Diagonalization algorithm combining with the Lenstra Lenstra-Lovász (LLL) lattice reduction technique to improve the quality of MU-MIMO systems. In the Zu's approach, the first precoding matrix is designed based on the QR decomposition while the second on is created by combining the linear precoding algorithms and the LLL method. Simulation results showed that the precoding algorithm significantly improves the system performance. However, the number of QR operations and the size of two precoding matrices in this proposal increase proportionally with the number of transmit antennas and the number of users. Therefore, the complexity of the algorithm is very large when it is applied to Massive MIMO systems.

Among various techniques, the transmit antenna selection is an important technique to enhance the system performance and energy efficiency. In addition, it reduces the number of radio frequency (RF) chains at the BS, and hence reducing the system cost and power consumption. In [13], the authors proposed the transmit antenna selection algorithm according to group based on the channel capacity analysis for Massive MIMO systems. Antenna groups that contribute the most to the total channel capacity will be selected. The channel capacity is calculated based on the singular value decomposition (SVD) operations of channel matrix. However, the complexity of this proposal is relatively high due to the SVD operations of channel matrix. In [10] and [14], the authors proposed the transmit antenna selection algorithm based on the channel capacity analysis for MIMO and Massive MIMO systems, respectively. In [15], the authors proposed the transmit antenna selection algorithm based on the norm analysis and the correlation between columns of the channel matrix to maximize energy efficiency for Massive MIMO system. In [16], based on the principal components analysis technique, the authors proposed the transmit antenna selection algorithm to remove antennas that contribute least to the total channel capacity for Massive MIMO system. The proposed algorithms in [10], [13], [14], [15] and [16] significantly improve the energy efficiency and performance of the system. However, these algorithms are performed by scanning the antennas in a one-by-one basis. Therefore, the selection complexity becomes very large as the number of antennas at the BS increases.

In this paper, we propose a transmit antenna selection aided linear group precoder for Massive MIMO systems. In the proposed method, a transmit antenna group selection algorithm based on the channel capacity analysis is implemented. To be specific, the channel matrix from the BS to all users is divided into $G$ groups, each of which consists of a number of the channel matrix's columns. The antenna groups that contribute the most to the total channel capacity will be used for signal transmission. Before being transmit to the user side via the selected antennas, the signal is precoded by a low complexity linear precoder, which is developed based on the conventional linear precoders in combination with the element-base lattice reduction shortest longest vector
(ELR-SLV) technique. To balance between the complexity and the system performance, the proposed precoder is designed to have two components: the first one minimizes the interference among neighboring user groups; the second improves the system performance by utilizing the ELR-SLV technique. Numerical and simulation results show that the system performance significantly improves when using the transmit selection technique. Moreover, by increasing the number of groups, the proposed precoder is capable of approach the BER performance of its LC-RBD-LR-ZF counterpart at remarkably lower computational complexities. However, it should be noted that increasing the number of groups will lead to higher precoding complexities.

The rest of this paper is organized as follows. In Section II, we present the Massive MIMO system model. The transmit antenna group selection algorithm is presented in Section III. In Section IV, we develop the linear group precoding algorithm combination with ELR-SLV technique. Simulation results are shown in Section V. Finally, conclusions are drawn in Section VI.

Notation: The notations are defined as follows: Matrices and vectors are represented by symbols in bold; $(.)^{T}$ and $(.)^{H}$ denote the transpose and conjugate transpose respectively. We reserve $|\alpha|$ for the absolute value of scalar $\alpha$ and $\operatorname{det}(\mathbf{B})$ for the determinant of B. $\lfloor\alpha\rfloor$ is to round down the real and imaginary parts of the complex number to the nearest integer, respectively. $\mathbf{I}_{N_{R}}$ denotes the $N_{R} \times N_{R}$ identity matrix. $\operatorname{Tr}\{$.$\} is$ the trace of a square matrix.

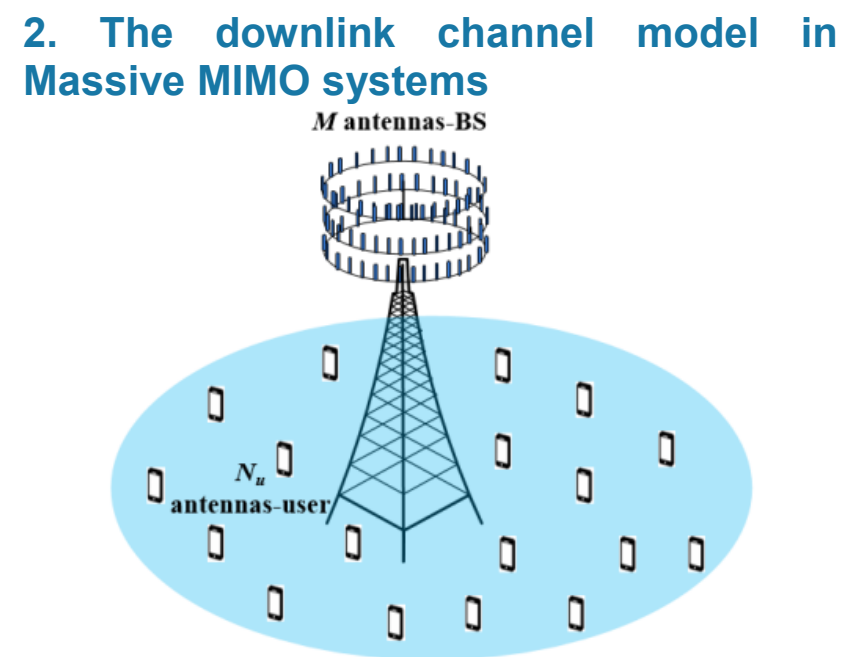

Figure 1. The downlink channel model in Massive MIMO system

Let us consider a Massive MIMO system having the BS equipped with $M$ antennas to simultaneously serve $K$ users as illustrated in Fig. 1. Each user is equipped with $N_{u}$ antennas. Thus, the total number of antennas for $K$ users is $N_{R}=K N_{u}$. 
In addition, the Channel State Information (CSI) is assumed to be perfectly known by the BS. Among $M$ antennas, $N_{T}$ antennas is selected at a time for signal transmission, where $N_{R} \leq N_{T} \leq M$.

Let $\mathbf{y} \in \square^{N_{R} \times 1}$ be the overall received signal vector for all users. Therefore, the relationship between the transmitted signal vector $\mathbf{x}=\left[\mathbf{x}_{1}^{T} \mathbf{x}_{2}^{T} \ldots \mathbf{x}_{K}^{T}\right]^{T} \in \square^{N_{R} \times 1}$ and the received signal vector $\mathrm{y}$ is represented by

$$
\mathbf{y}=\mathbf{H W x}+\mathbf{n},
$$

where $\mathbf{H} \in \square^{N_{R} \times N_{T}}$ is channel matrix from selected antennas at the BS to all $K$ users, whose entries are assumed to be independent and identically distributed (i.i.d) random variables with zero mean and unit variance; $\quad \mathbf{W}=\left[\mathbf{W}_{1} \mathbf{W}_{2} \ldots \mathbf{W}_{K}\right] \in \square^{N_{T} \times N_{R}}$ is the precoding matrix for all users; and $\mathbf{n}_{u} \in \square^{N_{R} \times 1}$ is noise vector at the all users, whose entries are assumed to be i.i.d random variables with zero mean and variance $\sigma^{2}$.

\section{Transmit antenna groups selection (TA-GS) algorithm in Massive MIMO system}

To balance the complexity and performance of the system, in this section, we propose the transmit antenna group selection algorithm based on the capacity analysis. The channel matrix from BS to all users is divided into $G\left(G=\left\lfloor N_{T} / \delta\right\rfloor\right)$ groups, $\quad \mathbf{H}_{g} \in \square^{N_{R} \times \delta}$, $(g=1,2, \ldots, G)$, where $\delta$ is a positive integer greater than 1. The channel matrix from $\mathrm{BS}$ to all users is expressed as

$$
\mathbf{H}_{M}=\left[\mathbf{H}_{1}, \mathbf{H}_{2}, \ldots, \mathbf{H}_{G}\right] .
$$

The first group, $\mathbf{H}_{1}$ includes $\delta$ first columns of the channel matrix; the second group $\mathbf{H}_{2}$ includes the $(\delta+$ 1)th to the $2 \delta$ th columns; and the last group, $\mathbf{H}_{G}$ includes the $(M-\delta)$ th to the $M$ th columns [13].

The first antenna group among $G$ antenna groups, that has the largest channel capacity is selected by the following expression

$$
\begin{aligned}
g_{1} & =\underset{g \in(1, \cdots, G)}{\operatorname{argmax}} C\{g\} \\
& =\underset{g \in(1, \cdots, G)}{\operatorname{argmax} \operatorname{mog}} \log _{2} \operatorname{det}\left[\mathbf{I}_{N_{R}}+\frac{\rho}{N_{T}} \mathbf{H}_{g} \mathbf{H}_{g}^{H}\right],
\end{aligned}
$$

herein, $\mathbf{H}_{g}$ is the $g$ th group of $\mathbf{H}_{M}, g$ is the index of the groups and $\rho$ is the average signal-to-noise ratio (SNR) at the receiver side.

After the first antenna group is selected, we combine each of the remaining $G-1$ antenna groups with $g_{1}$ to create $\mathbf{H}_{\left[g_{1}, g\right]}=\left[\mathbf{H}_{g_{1}}, \mathbf{H}_{g}\right] \in \square^{N_{R} \times 2 \delta}$. The second antenna group is selected such that the channel capacity provided by $g_{1}$ and itself is maximized. It is expressed as follows

$$
\begin{aligned}
g_{2} & =\arg \max _{g \in(1, \cdots, G), g \neq g_{1}} C\left\{g_{1}, g\right\} \\
& =\arg \max _{g \in(1, \cdots, G), g \neq g_{1}} \log _{2} \operatorname{det}(\mathbf{Q})
\end{aligned}
$$

where $\mathbf{Q}=\mathbf{I}_{N_{R}}+\frac{\rho}{N_{T}} \mathbf{H}_{\left[g_{1}, g\right]} \mathbf{H}_{\left[g_{1}, g\right]}^{H}$. The same process is carried out until $N_{T}$ transmit antennas are selected.

The proposed algorithm TA-GS is summarized in Algorithm 1.

Algorithm 1: Proposed TA-GS algorithm

1. Input $M, N_{T}, N_{R}, \delta, \mathbf{H}_{M} \in \square^{N_{R} \times M}$

2. Compute $G=\left\lfloor\frac{N_{T}}{\delta}\right\rfloor$ and create

$\mathbf{H}_{M}=\left[\mathbf{H}_{1}, \mathbf{H}_{2}, \ldots, \mathbf{H}_{G}\right]$.

3. Select the first antenna group according to:

$$
g_{1}=\underset{g \in(1, \cdots, G)}{\operatorname{argmax}} \log _{2} \operatorname{det}\left[\mathbf{I}_{N_{R}}+\frac{\rho}{N_{T}} \mathbf{H}_{g} \mathbf{H}_{g}^{H}\right] \text {. }
$$

4. Generate the channel matrix $\mathbf{H}_{\left[g_{1}, \cdots, g_{k-1}, g\right]}$ and select the $k$ th antenna group according to:

$$
\begin{aligned}
& g_{k}=\underset{g \in(1, \cdots, G), g \neq g_{1}, \cdots, g_{k-1}}{\arg } \operatorname{mag}_{2} \operatorname{det}(\mathbf{Q}), \text { where } \\
& \mathbf{Q}=\mathbf{H}_{\left[g_{1}, \cdots, g_{k-1}, g\right]} \mathbf{H}_{\left[g_{1}, \cdots, g_{k-1}, g\right]}^{H}
\end{aligned}
$$

5. Repeat Step 4 until $N_{T}$ transmit antennas are selected.

6. Output: $\mathbf{H} \in \square^{N_{R} \times N_{T}}$ 


\section{Proposed low-complexity lattice} reduction-aided linear group precoding (LR-LGP).

\subsection{Algorithm Analysis}

In this Section, a linear group precoding algorithm based on the linear precoding algorithms combination with the ELR-SLV technique, called LR-LGP, is proposed. The precoding matrix for all user groups is given by

$$
\mathbf{W}=\beta \mathbf{W}_{a} \mathbf{W}_{b} .
$$

In the first step, the channel matrix $\mathbf{H}$ is divided into $L\left(L=\frac{N_{R}}{\alpha}\right)$ groups (sub-matrices) $\mathbf{H}_{l} \in \square^{\alpha \times N_{T}}$, $(l=1,2, \ldots, L)$, where, $\alpha$ is a positive integer greater than 1. To be specific, the first group, $\mathbf{H}_{1}$, consists of the first row to the $\alpha$ th row of $\mathbf{H}$; the second group, $\mathbf{H}_{2}$, is from the $(\alpha+1)$ th row to the $2 \alpha$ th row; and the last group, $\mathbf{H}_{L}$, is from the $\left(N_{R}-\alpha\right)$ th row to the $N_{R}$ th row. Therefore, the channel matrix $\mathbf{H}$ can be rewritten as

$$
\mathbf{H}=\left[\begin{array}{l}
\mathbf{H}_{1} \\
\mathbf{H}_{2} \\
\vdots \\
\mathbf{H}_{L}
\end{array}\right] .
$$

In the second step, an MMSE weight matrix $\mathbf{W}_{\text {MMSE }} \in \square^{N_{T} \times N_{R}}$ for all users is constructed as follows

$$
\begin{aligned}
\mathbf{W}_{M M S E} & =\mathbf{H}^{H}\left(\mathbf{H H}^{H}+\sigma_{n}^{2} \mathbf{I}\right)^{-1} \\
& =\left[\mathbf{W}_{M M S E}^{G P_{1}}, \mathbf{W}_{M M S E}^{G P_{2}}, \ldots, \mathbf{W}_{M M S E}^{G P_{L}}\right],
\end{aligned}
$$

where $\sigma_{n}^{2}=\sigma^{2} / E_{s}, E_{s}$ is the symbol energy of the transmitted signals, $\mathbf{W}_{M M S E}^{G P_{l}} \in \square^{N_{T} \times \alpha}, l=1,2, \cdots, L$, are the MMSE weight matrix for the $l$ th user group.

Applying QR decomposition to $\mathbf{W}_{M M S E}^{G P_{I}}$, we get

$$
\mathbf{W}_{M M S E}^{G P_{l}}=\mathbf{Q}_{l} \mathbf{R}_{l},
$$

where, $\mathbf{Q}_{l} \in \square^{N_{T} \times N_{T}}$ is a unitary matrix with orthogonal columns, $\quad \mathbf{R}_{l} \in \square^{N_{T} \times \alpha}$ is an upper triangular matrix. We have

$$
\begin{aligned}
\mathbf{Q}_{l} \mathbf{R}_{l} & =\left[\begin{array}{ll}
\mathbf{Q}_{l, 1} & \mathbf{Q}_{l, 2}
\end{array}\right]\left[\begin{array}{l}
\mathbf{R}_{l, 1} \\
\mathbf{0}
\end{array}\right] \\
& =\mathbf{Q}_{l, 1} \mathbf{R}_{l, 1},
\end{aligned}
$$

here $\mathbf{Q}_{l, 1} \in \square^{N_{T} \times \alpha}, \mathbf{R}_{l, 1} \in \square^{\alpha \times \alpha}, \mathbf{Q}_{l, 2} \in \square^{N_{T} \times\left(N_{T}-\alpha\right)}$ and the zero matrix has size $\left(N_{T}-\alpha\right) \times \alpha$. Therefore, $\mathbf{W}_{a}^{G P_{l}}=\mathbf{Q}_{l, 1}$ is used as the precoding matrix for the $l$ th user group. Using $\mathbf{W}_{a}^{G P_{l}},(l=1, \cdots, L)$, the first precoding matrix $\mathbf{W}_{a} \in \square^{N_{T} \times N_{R}}$ is constructed as follows:

$$
\mathbf{W}_{a}=\left[\mathbf{W}_{a}^{G P_{1}}, \mathbf{W}_{a}^{G P_{2}}, \ldots, \mathbf{W}_{a}^{G P_{L}}\right] .
$$

In the next step, the second precoding matrix $\mathbf{W}_{b} \in \square^{N_{R} \times N_{R}}$ in (5) is created by combining the linear precoding algorithm and the lattice reduction technique to improve the quality of the system.

First, the effective channel matrix for the $l$ th user group is generated as follows

$$
\overline{\mathbf{H}}_{l}=\mathbf{H}_{l} \mathbf{W}_{a}^{G P_{l}},
$$

which is then used to generate the extended matrix $\overline{\mathbf{H}}_{l}^{\text {ext }} \in \square^{\alpha \times 2 \alpha}$ as follows

$$
\overline{\mathbf{H}}_{l}^{\text {ext }}=\left[\overline{\mathbf{H}}_{l}, \sqrt{\frac{N_{R} \sigma^{2}}{E_{s}}} \mathbf{I}_{\alpha}\right] .
$$

where $\mathbf{I}_{\alpha}$ is a unit matrix size $\alpha \times \alpha$.

Next, the channel matrix $\left(\overline{\mathbf{H}}_{l}^{\text {ext }}\right)^{T}$ is converted to the matrix $\hat{\mathbf{H}}_{l}$ in the LR domain by using the ELR-SLV algorithm in [17] to give

$$
\hat{\mathbf{H}}_{l}=\mathbf{U}_{l}^{T} \overline{\mathbf{H}}_{l}^{e x t}
$$

where $\mathbf{U}_{l}$ is a unimodular matrix with integer elements $\left(\operatorname{det}\left|\mathbf{U}_{l}\right|=1\right)$. After that, the precoding matrix $\mathbf{W}_{b}^{G P_{l}} \in \square^{\alpha \times \alpha}$ of the $l$ th user group is designed as follows

$$
\mathbf{W}_{b}^{G P_{l}}=\mathbf{A}_{l} \hat{\mathbf{H}}_{l}^{H}\left(\hat{\mathbf{H}}_{l} \hat{\mathbf{H}}_{l}^{H}\right)^{-1}
$$

where $\mathbf{A}_{l}=\left[\mathbf{I}_{\alpha}, \mathbf{0}_{\alpha}\right], \mathbf{0}_{\alpha}$ denotes the $\alpha \times \alpha$ zero matrix.

Finally, the precoding matrix $\mathbf{W}_{b}$ is constructed using $\mathbf{W}_{b}^{G P_{l}},(l=1, \cdots, L)$, as follows

$$
\mathbf{W}_{b}=\left[\begin{array}{llll}
\mathbf{W}_{b}^{G P_{1}} & 0 & \cdots & 0 \\
0 & \mathbf{W}_{b}^{G P_{2}} & \cdots & 0 \\
\vdots & \vdots & \ddots & \vdots \\
0 & 0 & & \mathbf{W}_{b}^{G P_{L}}
\end{array}\right] .
$$




$$
\mathbf{U}_{b}=\left[\begin{array}{llll}
\mathbf{U}_{1}^{T} & 0 & \cdots & 0 \\
0 & \mathbf{U}_{2}^{T} & \cdots & 0 \\
\vdots & \vdots & \ddots & \vdots \\
0 & 0 & & \mathbf{U}_{L}^{T}
\end{array}\right] .
$$

The normalized power factor is defined as

$$
\beta=\sqrt{\frac{N_{R}}{\operatorname{Tr}\left[\left(\mathbf{W}_{a} \mathbf{W}_{b}\right)\left(\mathbf{W}_{a} \mathbf{W}_{b}\right)^{H}\right]}} .
$$

The proposed algorithm LR-LGP is summarized in Algorithm 2.

\section{Algorithm 2: The LR-LGP precoding algorithm}

1. Input $N_{T}, N_{R}, \mathbf{H}$

2. Decide the number of user groups $L$ and compute the size of the sub-matrices.

3. Create the matrices $\mathbf{H}=\left[\mathbf{H}_{1}^{T}, \mathbf{H}_{2}^{T}, \ldots, \mathbf{H}_{L}^{T}\right]^{T}$ and $\mathbf{W}_{M M S E}=\left[\mathbf{W}_{M M S E}^{G P_{1}}, \mathbf{W}_{M M S E}^{G P_{2}}, \ldots, \mathbf{W}_{M M S E}^{G P_{L}}\right]$.

4. Apply $\mathrm{QR}$ decomposition to $\mathbf{W}_{M M S E}^{G P_{l}}$, i.e., $\mathbf{W}_{M M S E}^{G P_{l}}=\mathbf{Q}_{l} \mathbf{R}_{l}$ for $l=1, \cdots, L$.

5. Create the weight matrices: $\mathbf{W}_{a}^{G P_{l}}=\mathbf{Q}_{l}(:, 1: \alpha)$ for $l=1, \cdots, L$.

6. Create the weight matrix $\mathbf{W}_{a}$ by arranging $\mathbf{W}_{a}^{G P_{l}}$ as in equation (10).

7. Create the matrices $\overline{\mathbf{H}}_{l}=\mathbf{H}_{l} \mathbf{W}_{a}^{G P_{l}}$ and $\overline{\mathbf{H}}_{l}^{\text {ext }}=\left[\overline{\mathbf{H}}_{l}, \sqrt{\frac{N_{R} \sigma^{2}}{E_{s}}} \mathbf{I}_{G P}\right]$ for $l=1, \cdots, L$.

8. Convert $\left(\overline{\mathbf{H}}_{l}^{\text {ext }}\right)^{T}$ into $\hat{\mathbf{H}}_{l}$, for $l=1, \cdots, L$, by applying the ELR-SLV algorithm in [17].

9. Create the weight matrices $\mathbf{W}_{b}^{G P_{l}}=\mathbf{A}_{l} \hat{\mathbf{H}}_{l}^{H}\left(\hat{\mathbf{H}}_{l} \hat{\mathbf{H}}_{l}^{H}\right)^{-1}$ for $l=1, \cdots, L$.

10. Create the weight matrix $\mathbf{W}_{b}$ by arranging $\mathbf{W}_{b}^{G P_{l}}$, $l=1, \cdots, L$, as in (15).

11. Output: $\beta=\sqrt{\frac{N_{R}}{\operatorname{Tr}\left[\left(\mathbf{W}_{a} \mathbf{W}_{b}\right)\left(\mathbf{W}_{a} \mathbf{W}_{b}\right)^{H}\right]}}$ and $\mathbf{W}=\beta \mathbf{W}_{a} \mathbf{W}_{b}$.
The received signal vector for all users is given by

$$
\mathbf{y}=\mathbf{H W x}+\mathbf{n} .
$$

Using $\mathbf{y}$ in (18), the transmit signal vector is estimated as follows

$$
\hat{\mathbf{x}}=\mathbf{U}_{b} \frac{1}{\alpha}\left(Q_{z}\left[\alpha \frac{\mathbf{y}}{\beta}+\beta_{z}\left(\mathbf{U}_{b}\right)^{-1} \mathbf{1}_{L}\right]-\beta_{z}\left(\mathbf{U}_{b}\right)^{-1} \mathbf{1}_{L}\right),
$$

herein $\alpha=1 / 2, \beta_{z}=\frac{m-1}{2}(1+j), m$ is the number of bits in a transmitted symbol, $\mathbf{1}_{L} \in R^{N_{R} \times 1}$ is a column vector containing $N_{R}$ ones. $Q_{z}[$.$] represents the rounding$ operation. From (18) and (19), it follows that:

$$
\hat{\mathbf{x}}=\mathbf{x}+2 \mathbf{U}_{b} Q_{z}\left[\frac{1}{2} \frac{\mathbf{n}}{\beta}\right] .
$$

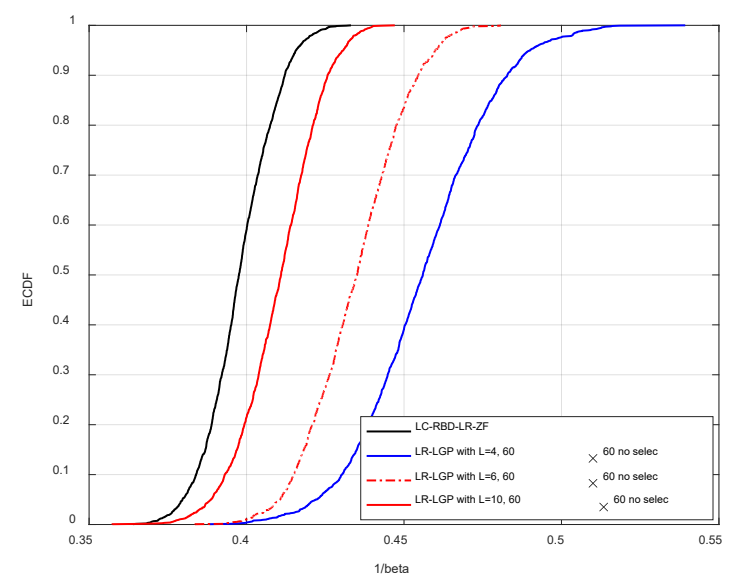

Figure 2. Empirical CDF of $1 / \beta$ for the LC-RBD-LRZF and LR-LGP precoders with $N_{T}=M=60$, $N_{u}=1, K=60, L=4,6$ and 10.

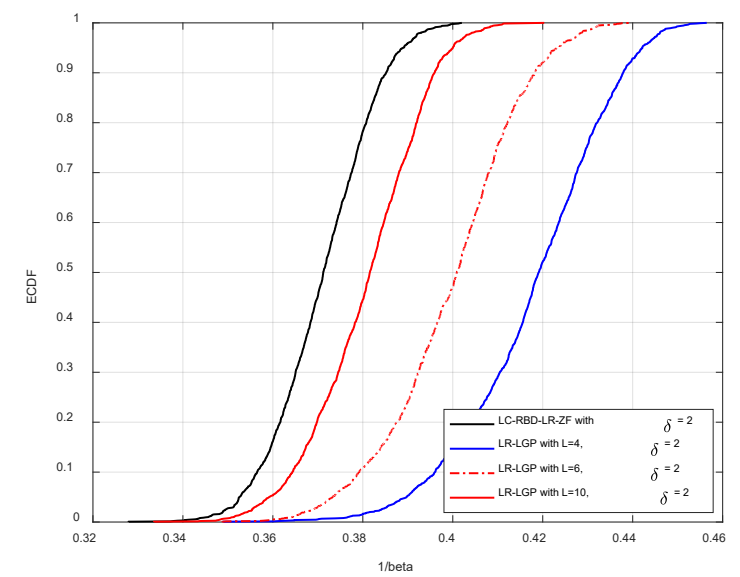

Figure 3. Empirical CDF of $1 / \beta$ for the LC-RBD-LRZF and LR-LGP precoders with $M=90, N_{T}=60$,

$$
N_{u}=1, K=60, L=4,6 \text { and } 10 .
$$


From (20), we can see that $\mathbf{x}$ is correctly recovered if $Q_{z}\left[\frac{1}{2} \frac{\mathbf{n}}{\beta}\right]=\mathbf{0}$. This means that for a given noise power, the component $1 / \beta$ will be the factor that determines the system performance. In Fig. 2 and Fig. 3, the empirical cumulative distribution functions (ECDFs) of the $1 / \beta$ in (20) are shown for the LC-RBD-LR-ZF and LR-LGP precoders when the system is with and without transmit antenna selection. For the same system configuration, the simulation results show that the LC-RBD-LR-ZF precoder generates smaller $1 / \beta$ than the LR-LGP precoder. This means that the LC-RBD-LRZF precoder will probably outperform the LR-LGP precoder for the same system configuration. Moreover, the more sub-groups are generated the smaller $1 / \beta$ becomes, and hence the better system performance can be achieved. It can also be observed from the two figures that the transmit antenna selection technique allows the system to reduce $1 / \beta$, thereby improving the system performance.

\subsection{Computational Complexity Analysis}

In this sub section, we evaluate the computational complexity of the proposed LR-LGP algorithm and the LC-RBD-LR-ZF algorithm in [12]. The complexities are evaluated by counting the required floating point operations (flops). We assume that each real operation (an addition, a multiplication or a division) is counted as a flop. Hence, a complex multiplication and a division equal to 6 flops and 11 flops, respectively. It is worth noting that the QR decomposition of an $r \times t$ complex matrix requires $6 r t^{2}+4 r t-t^{2}-t$ flops. Based on the above assumptions, the computational complexities of the proposed LR-LGP algorithm and the LC-RBD-LR-ZF one are computed in details as follows

\section{a. Complexity of the LC-RBD-LR-ZF algorithm}

The computational complexity of the LC-RBD-LR-ZF algorithm is given by:

$$
F=F_{a}+F_{b}+F_{c} \text { (flops). }
$$

herein $F_{a}$ and $F_{b}$ are the number of flops to calculate $\mathbf{P}_{a}$ and $\tilde{\mathbf{P}}_{b}$, respectively. $F_{c}$ is the number of flops of the multiplication two matrices $\mathbf{P}_{a}$ and $\tilde{\mathbf{P}}_{b} . F_{a}$ and $F_{b}$ are evaluated and expressed in the (22) and (23).

$$
\begin{aligned}
F_{a}= & K\left[6\left(N_{R}-N_{u}\right)\left(N_{R}+N_{T}-N_{u}\right)^{2}\right. \\
& +4\left(N_{R}-N_{u}\right)\left(N_{R}+N_{T}-N_{u}\right)-\left(N_{R}+N_{T}-N_{u}\right)^{2} \\
& \left.-\left(N_{R}+N_{T}-N_{u}\right)\right] \quad(\text { flops }) \\
F_{b}= & K\left(8 N_{T}^{2} N_{u}-2 N_{T} N_{u}\right)+K\left(16 N_{u}^{2} N_{T}-2 N_{u} N_{T}\right. \\
& \left.+8 N_{u}^{3}-2 N_{u}^{2}+F_{u p d a t e-L L L}\right)+K\left(8 N_{u}^{3}+16 N_{u}^{2} N_{T}\right. \\
& \left.-2 N_{u}^{2}-2 N_{u} N_{T}\right) \quad(\text { flops })
\end{aligned}
$$

Note that in (23), $F_{\text {update-LLL }}$ is the computational cost of the update operation for the LLL technique, which is obtained by computer simulation.

$F_{c}$ is evaluated to be

$$
F_{c}=8 K N_{T}^{2} N_{R}-2 N_{T} N_{R} \text { (flops). }
$$

From (21)-(24), the total number of flops of the LCRBD-LR-ZF algorithm is obtained as in (25).

$$
\begin{aligned}
F= & K\left[6\left(N_{R}-N_{u}\right)\left(N_{R}+N_{T}-N_{u}\right)^{2}\right. \\
& +4\left(N_{R}-N_{u}\right)\left(N_{R}+N_{T}-N_{u}\right)-\left(N_{R}+N_{T}-N_{u}\right)^{2} \\
& \left.-\left(N_{R}+N_{T}-N_{u}\right)\right]+K\left(8 N_{T}^{2} N_{u}-2 N_{T} N_{u}\right) \\
& +K\left(16 N_{u}^{2} N_{T}-2 N_{u} N_{T}+8 N_{u}^{3}-2 N_{u}^{2}+F_{\text {update }-L L L}\right) \\
& +K\left(8 N_{u}^{3}+16 N_{u}^{2} N_{T}-2 N_{u}^{2}-2 N_{u} N_{T}\right) \\
& +8 K N_{T}^{2} N_{R}-2 N_{T} N_{R} \quad(\text { flops }) \\
& \sim O\left(N_{R}^{4}\right)
\end{aligned}
$$

\section{b. Complexity of the LR-LGP algorithm}

The computational complexity of the proposed LRLGP algorithm is given by

$$
F_{1}=F_{A}+F_{B}+F_{C} \text { (flops). }
$$

where $F_{A}$ and $F_{B}$ are the number of flops to calculate $\mathbf{W}_{a}$ and $\mathbf{W}_{b}$, respectively. $F_{C}$ is the number of flops of multiplying the two matrices $\mathbf{W}_{a}$ and $\mathbf{W}_{b}$.

$F_{A}$ is expressed as

$$
F_{A}=F_{M M S E}+F_{Q R} \text { (flops), }
$$

where $F_{M M S E}$ is the number of flops to compute the $\mathbf{W}_{M M S E}$ matrix and $F_{Q R}$ is number of flops for the QR 
decomposition operations. $F_{M M S E}$ and $F_{Q R}$ are calculated as follows

$$
\begin{gathered}
F_{\text {MMSE }}=8 N_{R}^{3}+16 N_{R}^{2} N_{T}-N_{R}^{2} \\
-2 N_{R} N_{T}+N_{R}+1 \quad(\text { flops }) . \\
F_{Q R}=L\left(6 N_{T}^{2} \alpha+4 N_{T} \alpha-\alpha^{2}-\alpha\right)(\text { flops }) .
\end{gathered}
$$

Therefore, the total number of flops to find $\mathbf{W}_{a}$ is given by

$$
\begin{aligned}
F_{A} & =F_{M M S E}+F_{Q R} \\
& =8 N_{R}^{3}+16 N_{R}^{2} N_{T}-N_{R}^{2}-2 N_{R} N_{T}+N_{R} \\
& +1+L\left(6 N_{T}^{2} \alpha+4 N_{T} \alpha-\alpha^{2}-\alpha\right)(\text { flops }) .
\end{aligned}
$$

$F_{B}$ is calculated as follows

$$
F_{B}=F_{2}+F_{3}+F_{4} \text { (flops), }
$$

where $F_{2}, F_{3}$ and $F_{4}$ are the total number of flops to compute $\overline{\mathbf{H}}_{l}, \hat{\mathbf{H}}_{l}$ and $\mathbf{W}_{b}^{G P_{l}}$ matrices, respectively. $F_{2}$ and $F_{3}$ are computed as

$$
\begin{aligned}
& F_{2}=L\left(8 N_{T} \alpha^{2}-2 \alpha^{2}\right) \quad(\text { flops }) . \\
& \left.F_{3}=F_{5}+F_{6}+F_{\text {update-SLV }} \quad \text { (flops }\right) .
\end{aligned}
$$

herein $F_{5}$ and $F_{6}$ are the number of flops to compute

$$
\tilde{\mathbf{C}}=\left[\left\{\left(\overline{\mathbf{H}}_{l}^{e x t}\right)^{T}\right\}^{H}\left(\overline{\mathbf{H}}_{l}^{e x t}\right)^{T}\right]^{-1} \quad \text { and } \quad \hat{\mathbf{H}}_{l}=\mathbf{U}_{l}^{T} \overline{\mathbf{H}}_{l}^{e x t},
$$

respectively. $F_{\text {update-SLV }}$ is computational cost of the ELRSLV algorithm's update operations, which is obtained by computer simulation. Therefore, $F_{3}$ is calculated as

$$
\begin{array}{r}
F_{3}=L\left(24 \alpha^{3}-2 \alpha^{2}\right)+L\left(16 \alpha^{3}-4 \alpha^{2}\right) \\
+L F_{\text {update }-S L V} \quad(\text { flops }) .
\end{array}
$$

The number of flops to find all $\mathbf{W}_{b}^{G P_{l}}$ matrices is given by

$$
F_{4}=L\left(56 \alpha^{3}-8 \alpha^{2}\right) \quad \text { (flops). }
$$

Hence, the total number of flops to find the precoding matrix $\mathbf{W}_{b}$ is represented by

$$
\begin{aligned}
F_{B}= & L\left(8 N_{T} \alpha^{2}-2 \alpha^{2}\right)+L\left(24 \alpha^{3}-2 \alpha^{2}\right)+L\left(16 \alpha^{3}\right. \\
& \left.-4 \alpha^{2}\right)+L F_{\text {update }-S L V}+L\left(56 \alpha^{3}-8 \alpha^{2}\right)(\text { flops }) .
\end{aligned}
$$

$F_{C}$ is calculated by

$$
\left.F_{C}=8 N_{R}^{2} N_{T}-2 N_{R}^{2} \quad \text { (flops }\right)
$$

From the above analysis results, the total number of flops for the LR-LGP algorithm is given in the (38)

$$
\begin{aligned}
F_{1} & =8 N_{R}^{3}+16 N_{R}^{2} N_{T}-N_{R}^{2}-2 N_{R} N_{T}+N_{R}+1 \\
& +L\left(6 N_{T}^{2} \alpha+4 N_{T} \alpha-\alpha^{2}-\alpha\right)+L\left(8 N_{T} \alpha^{2}-2 \alpha^{2}\right) \\
& +L\left(24 \alpha^{3}-2 \alpha^{2}\right)+L\left(16 \alpha^{3}-4 \alpha^{2}\right)+L F_{\text {update }-S L V} \\
& \left.+L\left(56 \alpha^{3}-8 \alpha^{2}\right)+8 N_{R}^{2} N_{T}-2 N_{R}^{2} \text { (flops }\right) . \\
& \sim O\left(N_{R}^{3}\right)
\end{aligned}
$$

\section{Simulation results}

In this section, we evaluate and compare not only the BER performance but also the computational complexity of the LR-LGP precoder with those of the LC-RBD-LR$\mathrm{ZF}$ precoder in [12]. The channel between the BS and all users are assumed to be semi-static Rayleigh fading.

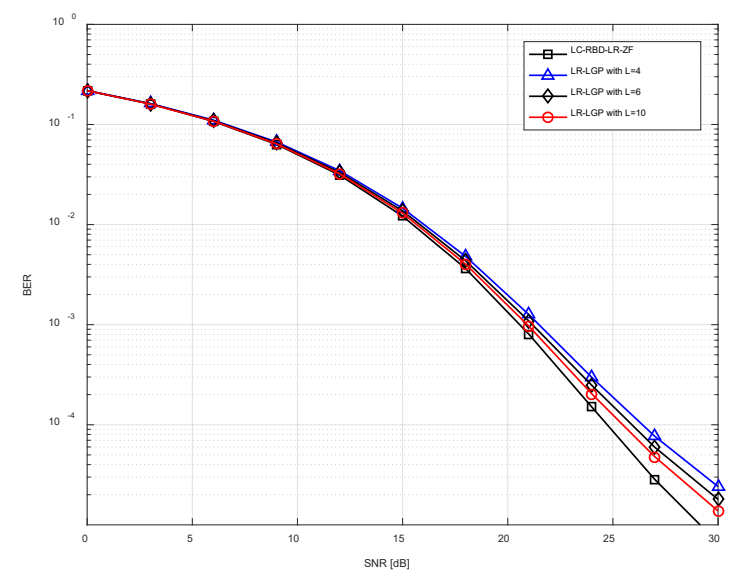

Figure 4. The system performance with $N_{T}=M=60$,

$$
N_{u}=1, K=60, L=4,6,10 .
$$

Fig. 4 illustrates the system performance for the proposed algorithm LR-LGP and the LC-RBD-LR-ZF algorithm in [12]. The simulation parameters are as follows: $N_{T}=M=60, \quad N_{u}=1, K=60$ and 4-QAM modulation. The number of user groups for the LR-LGP precoder is $L=4 ; 6$, and 10 . The simulation results in Fig. 4 show that the BER performance of the proposed algorithm LR-LGP is asymptotic to the LCRBD-LR-ZF algorithm when $L$ increases. Specifically, at $\mathrm{BER}=10^{-3}$ the proposed algorithm suffers from performance degradations of around $0.5 \mathrm{~dB}, 0.8 \mathrm{~dB}$ and $1.1 \mathrm{~dB}$ in SNR corresponding to $L=10 ; 6$ and 4 when compared to the LC-RBD-LR-ZF algorithm. However, the computational complexity of the proposed algorithm 
is significantly lower than the LC-RBD-LR-ZF algorithm, as confirmed by the simulation results in Fig. 7.

Fig. 5 and Fig. 6 illustrate the system performance when the TA-GS technique is applied. To get the results, the following parameters are used: $M=90$, $N_{T}=60, N_{u}=1, K=60, L=4,6,10,4-\mathrm{QAM}$ modulation, $\delta=2$ for Fig. 5 and $\delta=3$ for Fig. 6. It can be seen from the figures that the system performance is significantly improved as the TA-GS technique is adopted. Specifically, at $\mathrm{BER}=10^{-3}$ for the same precoder, the system performance improves by about $2 \mathrm{~dB}$ and $1.5 \mathrm{~dB}$ in SNR corresponding to $\delta=2$ and $\delta=3$ when compared to the case of no antenna selection. Besides, Fig. 5 and Fig. 6 show that the performance improvement is inversely proportional to $\delta$.

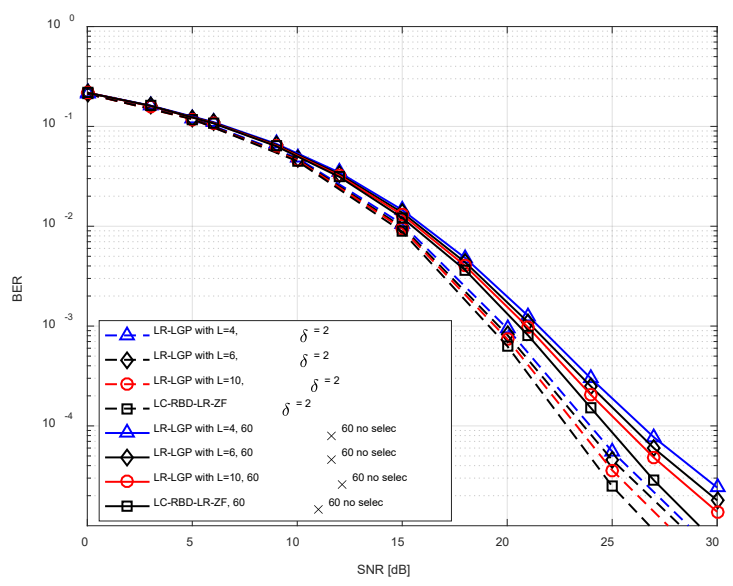

Figure 5. The system performance with $M=90$, $N_{T}=60, N_{u}=1, K=60, L=4,6,10, \delta=2$

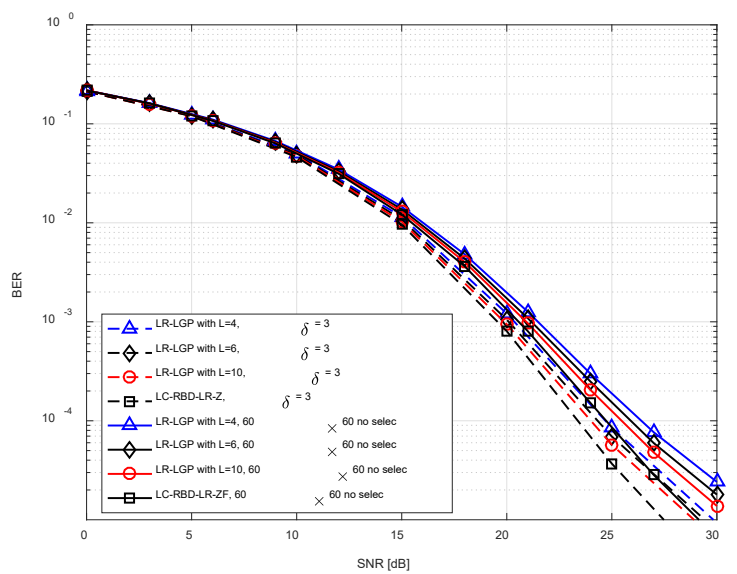

Figure 6. The system performance with $M=90$, $N_{T}=60, N_{u}=1, K=60, L=4,6,10, \delta=3$
Fig. 7 illustrate the computational complexities of the proposed algorithm LR-LGP and the LC-RBD-LR-ZF algorithm. In this scenario, $N_{T}$ is varied from 40 to 100 transmit antennas, $N_{R}=N_{T}, L=4$ and $L=10$. It can be seen from the figure that the computational complexities of the proposed precoder is noticeably lower than those of the LC-RBD-LR-ZF precoder. Specifically, at $N_{T}=80$ antennas, the computational complexities of the LR-LGP precoder with $L=4$ and $L=10$ groups are equal to about $6.25 \%, 13.54 \%$ of the LC-RBD-LR-ZF precoder's complexity, respectively. We can also see from the figure that the computational complexity of the LR-LGP precoder increases as the number of groups $L$ increases.

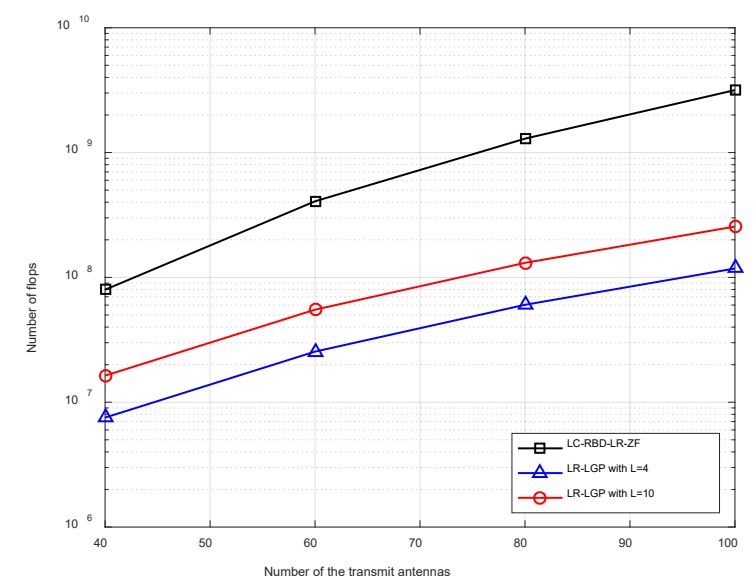

Figure 7. Compare the complexity of the proposed precoding algorithm and the LC-RBD-LR-ZF algorithm in [12]

\section{Conclusions}

In this paper, we propose the transmit antenna group selection technique for Massive MIMO system. The algorithm is performed on the basis of analyzing the channel capacity. Antenna groups that contribute the most to the total channel capacity will be selected. To trade the computational complexity off against the system performance, from the selected antennas, we develop a low-complexity lattice reduction-aided linear group precoding in Massive MIMO system, called LR-LPG precoder. The proposed precoder is developed based on the conventional linear precoder in combination with the ELR-SLV technique that has low complexity. Numerical and simulation results show that 
the system performance significantly improves when applying the transmit selection technique. Simulation results also show that the system performance inversely proportional to $\delta$. Furthermore, the LR-LGP precoder has remarkably lower complexity than the LC-RBD-LR$\mathrm{ZF}$, whereas its BER performance approaches that of the LC-RBD-LR-ZF precoder when $L$ increases. As a consequence, the LR-LGP can be a potential candidate for signal beamforming at the BS of Massive MIMO systems.

Acknowledgement: This paper has been presented in part at International Conference on Advanced Technologies for Communications, ATC, 2018

\section{References}

[1] H. Q. Ngo, Massive MIMO: Fundamentals and system designs. Linkoping University Electronic Press, 2015, vol. 1642

[2] Q. H. Spencer, C. B. Peel, A. L. Swindlehurst, and M. Haardt, "An introduction to the multi-user mimo downlink," IEEE Communications Magazine, vol. 42, no. 10, pp. 60-67, Oct 2004.

[3] L. Lu, G. Y. Li, A. L. Swindlehurst, A. Ashikhmin, and R. Zhang, "An overview of massive mimo: Benefits and challenges," IEEE Journal of Selected Topics in Signal Processing, vol. 8, no. 5, pp. 742-758, Oct 2014

[4] T. L. Marzetta, "Noncooperative cellular wireless with unlimited numbers of base station antennas," IEEE Transactions on Wireless Communications, vol. 9, no. 11, pp. 3590-3600, November 2010.

[5] - "Massive mimo: An introduction," Bell Labs Technical Journal, vol. 20, pp. 11-22, 2015.

[6] H. Y. T. L. Marzetta, E. G. Larsson and H. Q. Ngo, Fundamentals of Massive MIMO. Cambridge University Press, 2016.

[7] E. G. Larsson, O. Edfors, F. Tufvesson, and T. L. Marzetta, "Massive mimo for next generation wireless systems," IEEE Communications Magazine, vol. 52, no. 2, pp. 186-195, February 2014.

[8] V. P. Selvan, M. S. Iqbal, and H. S. Al-Raweshidy, "Performance analysis of linear precoding schemes for very large multi-user mimo downlink system," Fourth edition of the International Conference on the Innovative Computing Technology (INTECH 2014), pp. 219-224, Aug 2014.

[9] H. Q. Ngo, E. G. Larsson, and T. L. Marzetta, "Massive mu-mimo downlink tdd systems with linear precoding and downlink pilots," pp. 293-298, 2013.

[10] Y. S. Cho, J. Kim, W. Y. Yang, and C. G. Kang, MIMOOFDM wireless communications with MATLAB. John Wiley \& Sons, 2010.

[11] M. . Simarro, F. Domene, F. J. Martínez-Zaldívar, and A. Gonzalez, "Block diagonalization aided precoding algorithm for large mu-mimo systems," 2017 13th International Wireless Communications and Mobile
Computing Conference (IWCMC), pp. 576-581, June 2017.

[12] K. $\mathrm{Zu}$ and R. C. d. Lamare, "Low-complexity lattice reductionaided regularized block diagonalization for mumimo systems," IEEE Communications Letters, vol. 16, no. 6, pp. 925-928, June 2012.

[13] V. K. Dinh, M. Tuan Le, V. D. Ngo, and C. Hieu Ta, "Transmit antenna selection by group combination precoding in massive mimo system," 2018 International Conference on Advanced Technologies for Communications (ATC), pp. 276-281, Oct 2018.

[14] W. S. Bing Fang, Zuping Qian and W. Zhong, "Raise: A new fast transmit antenna selection algorithm for massive mimo systems," Springer Science+Business Media New York, September 2014.

[15] T. Tai, W. Chung, and T. Lee, "A low complexity antenna selection algorithm for energy efficiency in massive mimo systems," 2015 IEEE International Conference on Data Science and Data Intensive Systems, pp. 284-289, Dec 2015.

[16] M. T. A. Rana, R. Vesilo, and I. B. Collings, "Antenna selection in massive mimo using non-central principal component analysis," 2016 26th International Telecommunication Networks and Applications Conference (ITNAC), pp. 283-288, Dec 2016.

[17] Q. Zhou and X. Ma, "Element-based lattice reduction algorithms for large mimo detection," IEEE Journal on Selected Areas in Communications, vol. 31, no. 2, pp. 274286, February 2013. 